\title{
A Model of Two-Zoned Networks for Platform-Mediated Markets: Theory and Practical Applications
}

\author{
Ziru Li \\ Arizona State University \\ ziruli@asu.edu
}

\author{
Benjamin B. M. Shao \\ Arizona State University \\ Benjamin.Shao@asu.edu
}

\author{
Michael Goul \\ Arizona State University \\ Michael.Goul@asu.edu
}

\begin{abstract}
Two-sided networks enabled by information technology (IT) represent an emerging type of platform-mediated market in the digital economy. Researchers have studied the associated economic and strategic issues from both theoretical and managerial perspectives; however, we have noticed inadequacy in the extant literature when observing some real-world cases that cannot be fully explained by the framework of two-sided networks. A more comprehensive model is needed to explicate the broader market structure and understand the underlying market dynamics. To achieve this aim, we propose a theoretical model of two-zoned networks. In extending the existing dimension of "side," we add the "zone" dimension at a higher level to study two-zoned networks, which include two-sided networks as a special case. In the proposed model, two-zoned networks consist of two, two-sided networks and a core platform that serves both networks as their connection point at the nexus of two zones. Using the proposed model of two-zoned networks, we analyze four real-world business cases to demonstrate the model's practical applications. Finally, strategic implications of this model, in terms of operational and legal ramifications, are described. We conclude with topics for future research.
\end{abstract}

\section{Introduction}

Two-sided networks (2SN), also referred to as "two-sided markets" (2SM), "two-sided platforms" (2SP) and "platform-mediated networks" (PMN), have been described as a new market structure that accelerated the pace and impact of business transformation for firms across many industry sectors [5, 14, 15]. Electric utilities, financial services, healthcare, media, semiconductors, software, technology, telecommunications, and transportation have all experienced transformation associated with this new market structure. Two-sided networks consist of a core platform, sponsors, and multiple groups of participants such as suppliers, producers, intermediaries, customers and complementors [1]. The core platform provides the infrastructure, services, and rules to facilitate the connection, communication, and transactions among network users.

In this paper, we propose a theoretical model of two-zoned networks (2ZN) to extend the model of $2 \mathrm{SN}$. In each zone, there is a separate $2 \mathrm{SN}$ and the nexus of the two zones is a common, shared core platform that connects the two 2SN markets. The core platform provides both free and paid services, which leads to the labeling of the two zones as the free zone and the pay zone, respectively. Charging, however, is only one of many differences that distinguish the two zones; here free and pay zones are named solely for the sake of reference. We also emphasize two other significant differences between the zones: 1) end users being served can have different requirements and expectations between these two zones, and 2) the ways users interact with each other can differ in each zone. More important, these different mechanisms lead to different $2 \mathrm{SN}$ even while the core platform is still the same and can be shared. In each zone and between the two zones, there may exist same-side, cross-side, and cross-zone network effects. It is noted that the proposed 2ZN model differs from the freemium business model sometimes used for $2 \mathrm{SN}$. Freemium is a pricing model based on versioning, and it enables monetizing the same user group [14]. The new $2 \mathrm{ZN}$ model can help analyze platform competition more accurately and in more detail, and hence can help platform owners make more informed decisions.

In addition, we propose three attributes of twozoned networks: 1) zone parallelism, 2) service diversity in the pay zone, and 3) latent payment in the free zone. The level of functional similarity between the services offered in the free zone and those in the pay zone is called zone parallelism. Such similarity may manifest in the interactions of user groups, the functional taxonomy of the two-sided markets in each zone, and direct and indirect network effects. We then apply our model of two-zoned networks to four popular platforms to demonstrate its constructs and applicability. In doing so, we show that the new $2 \mathrm{ZN}$ 
model can extend existing conceptualizations prevalent in the extant literature.

One area of direct applicability for $2 \mathrm{ZN}$ is in legal contexts. Market structure plays a central role in analyzing antitrust issues [10]. The $2 \mathrm{ZN}$ model we propose can enlighten legal analysis of antitrust issues because it advances the understanding of market structure with consideration of the underlying features and attributes of multiple zones. For instance, the tests for small but significant and non-transitory increases in price (SSNIP) and for identifying a hypothetical monopolist (HM) have traditionally been used to analyze market power for monopoly [11]. However, Hoppner [8] and others note that when applied to twosided networks, those tests need to be either reinterpreted or modified as the cross-side indirect network effects are ignored but crucial to the assessment of market power and the conduct of a platform operator. With the two-sided network, particularly when one side charges zero prices by leveraging indirect network effects, the SSNIP test does not work well. Our $2 \mathrm{ZN}$ model has the potential to complement these existing antitrust tests by specifically pointing out the existence of "zone" beyond "side." Katz and Sallet [9] argue that multisided platforms do not require a new antitrust law; instead, regulators should apply existing principles with due diligence in assessing whether a particular firm has substantial market power to harm competition before any litigation is raised. Moreover, "profitability must be taken at a platform level and not on sales to just one side of the market," which implies the approaches to antitrust in platform markets need to extend beyond sides [13]. The proposed 2ZN model provides important insights into this topic. That importance is increasing today as governmental scrutiny of high-tech platforms for potential antitrust violations is being demanded by many stakeholders.

The remainder of the paper is organized as follows. In the next section, we review the extant literature of platform-mediated networks and network effects. We then elaborate the model of two-zoned networks. After that, we provide illustrative examples of two-zoned network markets and explicate how they differ from traditional two-sided network markets. We identify three essential attributes of two-zoned networks to reexamine these examples in Section 5. Finally, research and practical implications are discussed before we conclude the paper.

\section{Literature Review}

Platform-mediated networks have emerged as a prominent market structure, and there is a rich body of literature on this topic (e.g., [6]). Our literature review is not meant to be exhaustive but focused on the traditional framework of two-sided markets that Eisenmann [5] develops. The notion of platforms was initially introduced as "two-sided markets" [5, 16], which consist of platform provider, platform sponsor, component suppliers, and two or more groups of users. As shown in Figure 1, the core of the network is an intermediary platform that connects two distinct groups of users and facilitates their interactions for product and service exchanges. Transactions in a two-sided network entail a triangular set of relationships. The interactions between the two distinct user groups are subject to the cross-side (indirect) network effects.

Platforms need to address several critical issues in order to prevail in the fierce competition of two-sided network markets [5]. Several studies have focused on how to attract multiple sides to a platform [7]. In the process, pricing is one of the most important issues in platform development [3]. Because of the distinctive structure of $2 \mathrm{SN}$, pricing on such platforms is complicated. One essential decision is made in regards which side to subsidize and which side to charge [2]. The prerequisite for subsidization is that the platform can exploit and capture cross-side network effects. Essentially, a platform should subsidize the side that is more price-sensitive and charge the side that highly values cross-side interactions [14]. When making the pricing decision, a platform owner should consider other factors, such as output costs, same-side network effects, and brand value.

The literature of two-sided network markets is rich. However, we observe a gap in the literature because some business cases cannot be fully explained by the 2SN framework, particularly those where the free service part and pay service part involve different interaction mechanisms. Besides, several recent works also point out the necessity for more complex models $[4,12]$. Significant insights can be derived from analyzing group interactions, network effects, and mechanisms within different zones. We extend the existing research by creating a model of two-zoned networks to analyze these new features.

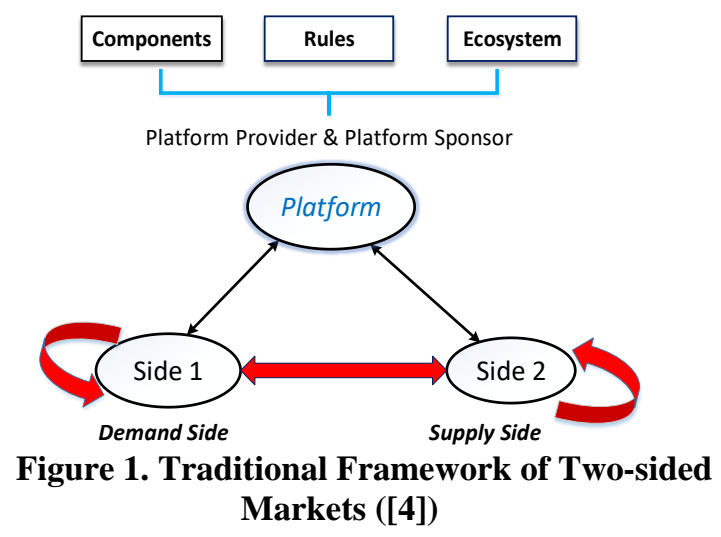




\section{Model of Two-Zoned Networks}

In two-sided networks, the platform plays one role, the mechanism between the platform and different user groups remains stable, and the strategies a platform adopts are relatively straightforward [5]. However, it is our observation that some real-world platforms have structures that are more complex. By analyzing their characteristics, we identify a need to extend two-sided networks (2SN) to two-zoned networks (2ZN).

The kernel of the traditional 2SN market is the mechanism through which the platform connects two distinct user groups. In other words, one single mechanism facilitates the interaction of users. From a user perspective, we refer to the usage of this mechanism as the "function" of the platform [5]. The 2SN framework indicates four categories of such functional use: connectivity, price-setting, variety, and matching. For example, eBay is a price-setting $2 \mathrm{SN}$ platform for online auctions that helps buyers with needs and sellers with offerings to find each other and decide transaction prices through bidding. On the other hand, Facebook, which derives $85 \%$ of its revenues from advertising, is a variety platform of social media. It attracts one side (viewers) that is subsidized by the other side (advertisers). Since the social network platform is subject to strong network effects, once the virtuous cycle was triggered, Facebook soon took a lion's share of the social media market.

While the framework of two-sided networks is useful, we observe some cases that cannot be fully explained by this model. Boingo, YouTube, LinkedIn, and GitHub all provide both free accounts and premium pay accounts, and it is the distinction between free accounts and pay accounts - as well as the differences in their functions - from which we derive our two-zoned network model. LinkedIn's premium account users use the platform primarily for recruiting and job-hunting purposes (i.e., matching), while free account holders use it mainly as an online social platform to network with other professionals (i.e., connectivity) and share content (i.e., variety). For free and paid users, the LinkedIn platform provides different value and facilitates the interaction of users within and between distinct groups through different mechanisms. As a result, we find it necessary to extend and enhance the traditional framework of two-sided networks. Specifically, we find that whether the provided service is free or not can be a deciding factor for differentiating these platforms from traditional ones. The reason is that the core platform mechanism fulfills different functions by providing different services for free vs. premium account users. Hence, the value creation strategies are different. Technically, we extend the traditional framework by adding the "zone" dimension at a higher level of abstraction above the "side" dimension. In each zone, customers use the platform and its provided services differently.

Figure 2 shows our proposed model of a two-zoned network. We define a two-zoned network as a platform-mediated network that encompasses both a free zone and a pay zone, each of which consists of a two-sided network. At the nexus of the two zones, the core platform serves as an intermediary that connects multiple groups of users. The core platform may play a different role in each zone by performing a different function. Following Eisenmann's [5] definitions for a two-sided network, platform sponsors are also called platform owners. Owners decide the rules, policies and standards for the platform. They can modify the platform's infrastructure technology and decide who can participate in the network and in which role. Platform providers connect distinct groups of users and mediate their interactions. They are the primary point of contact for users if there are any problems with using the platform. Platform component suppliers provide users with additional goods and services called platform components that are not directly available from platform providers. These complementary components can enhance user experience in using the platform. Demand-side users are also called end users. In the free zone, end users are not monetarily charged for using the platform. The services the platform provides fall under the "attraction" part. The goal of the free zone is to build a large user base quickly. In the pay zone, signing up for extended services requires users' monetary payments and thus relates to the "extraction" part. Users often face the same-side (direct) and cross-side (indirect) network effects.

From the perspective of platform providers, they may enjoy demand-side economies of scale, owing to positive network effects. Oftentimes, the user base that the platform has accumulated in the free zone can be harvested and monetized. Supply-side users, on the other hand, offer content as complements that are delivered through the core platform to be enjoyed by demand-side users. These content providers have interactions with demand-side users in each corresponding zone. In the "attraction" (free) zone, the goal is to grow the user base and hence the infrastructures and mechanisms are designed to quickly achieve a critical mass level necessary for the ignition of network effects. In the "extraction" (pay) zone, the goal instead is to generate profits, and the platform offers services to achieve this aim.

It is noted that a user group's role in one zone does not necessarily translate into the same role in the other zone. Advertisers, for example, may interact with users in the free zone as a platform requires free users to watch a short video commercial before they are 
allowed to use the free services (e.g., Wi-Fi or access to the requested content). However, once users sign up for a premium pay account, they would be excused from advertising. In Figure 2, the arc arrows in different colors indicate network effects, including red for the same-side (direct) network effects and green for the cross-side (indirect) network effects. The yellow straight arrow stands for communication between free users and paid users across zones and thus represents possible cross-zone network effects that are not examined in the $2 \mathrm{SN}$ literature. In each zone, the platform connects two (or more) user groups.
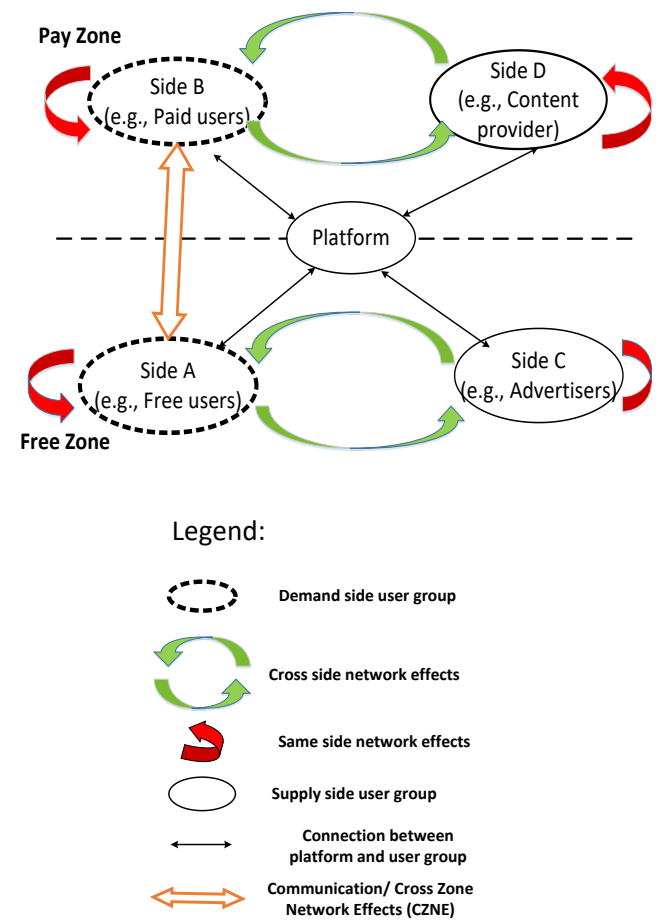

Figure 2. Model of Two-zoned Networks

Using structural taxonomy, Eisenmann [5] classifies two-sided networks into four functional categories: connectivity, variety, matching, and pricesetting. Therefore, the two-sided network in each zone of Figure 2 can belong to one such category. If the two 2SNs in both zones belong to the same functional category, this similarity is called "zone parallelism." In other words, the two-zoned network is said to have high zone parallelism if both of the two-sided markets perform the same function. On the other hand, the twozoned network shows low zone parallelism if the core platform plays a different role in each zone. In this case, the two 2SNs perform different functions in each zone, and the network effects within and between user groups in each zone may be quite different.

In the free zone, the platform may require users to make a latent payment, such as watching a short video commercial or revealing personal data (e.g., email address or physical location) prior to being allowed to use the free services. Whether users in the free zone have to make such a latent payment is another attribute of a two-zoned network that differentiates the new $2 \mathrm{ZN}$ model from the $2 \mathrm{SN}$ model. The latent payment is an embodiment of opportunity cost borne by free users, and if it exists, the no-cost services they access are not truly free. Nonetheless, the services offered in the free zone still fall under the "attraction" set.

On the other hand, in the pay zone, users are charged a fee to use additional services provided by the platform. Sometimes the paid services are an extended version of the basic free services but with a larger capacity and/or for a longer period of time. At other times, the platform offers completely new services because the targeted customers in the pay zone have different utility requirements from those in the free zone. In either case, these services belong to the "extraction" set. As a result, two-zoned networks may also vary in the diversity of services offered in the pay zone. Here we emphasize that zone parallelism is different from service diversity. Service diversity is a characteristic of the pay zone, while zone parallelism is an outcome characteristic based on direct comparison of the free zone and the pay zone. Oftentimes, the more diverse the pay zone is, the less similar the two zones are. However, at other times, service diversity can be low when zone parallelism is also low for a two-zoned network. In this situation, the services the platform offers in the pay zone perform the same function, but they still differ from those offered in the free zone. If service diversity is high, it means that the platform performs different functions, which in turn requires more complex coding efforts and advanced programmer IT skill sets. If service diversity is low, the platform can adopt the same infrastructure for the same functions. In this light, the implications of $2 \mathrm{ZN}$ for technical complexity and development cost are consistent with the logic of service computing.

In conclusion, the three factors - zone parallelism, latent payment in the free zone, and diversity of paid services in the pay zone - are identified as a triad of essential attributes in analyzing a two-zoned network. These factors make the new $2 \mathrm{ZN}$ model related to, but also distinct from, the $2 \mathrm{SN}$ model. With the additional level of "zone," we distinguish the free and paid services into two zones and separately analyze their underlying mechanisms. Different mechanisms can lead to different pricing strategies, budget planning, infrastructures, and IT investment requirements. This new model of two-zoned network can help platform owners better understand the mechanisms and make more informed decisions accordingly. In addition, these different mechanisms can be leveraged to more 
transparently demonstrate when there may be overt antitrust/monopolistic activities. In the next section, we apply the new model of two-zoned network to four real-world cases for comparison, and we analyze how they vary along dimensions based on the three factors.

\section{Practical Applications of the Two-zoned Network}

In this section, we discuss four specific platforms and apply our $2 \mathrm{ZN}$ approach to each for analysis. The first one is Boingo, a U.S.-based company that provides mobile Internet access for wireless-enabled consumer devices. We divide the Boingo platform into the free zone and the pay zone, as shown in Figure 3. Boingo customers do not interact with each other directly, as their primary goal is to access the Internet via Boingo's Wi-Fi services. Instead, their interactions on the platform are with the other group: advertisers in the free zone and the Internet service providers at the locations in the pay zone.

The core platform plays different roles in each of the two zones, where the two types of users are separated and served differently. Users in the free zone are required to watch advertising videos before being able to access free Wi-Fi for a limited period. Thus, in the free zone, Boingo generates revenues from the advertisers who, in essence, subsidize the free users. Boingo's Wi-Fi service falls into the "attraction" part of the service computing paradigm. There is a positive cross-side network effect from free users to advertisers: the more users it has, the more willing advertisers are to run their ads on the Boingo platform. In the free zone, advertisers are on the monetized side and the users are on the subsidized side. Customers in the free zone make latent payments via the time they spend watching the commercials. In this light, insights from using the $2 \mathrm{ZN}$ model can be generated into any scenario that Boingo may face with regard to pricing competition, tipping strategies, antitrust concern, etc.

In the pay zone, users pay a monthly subscription fee for a Wi-Fi plan and therefore do not need to watch any commercials. Boingo offers different Wi-Fi plans with varying numbers of devices allowed, and there may be different prices for different locations. The number and diversity of locations are the main factors that paid users consider when deciding whether to purchase Boingo's Wi-Fi services. In order to make its services attractive, the company must maintain a sufficient number of hotspots in diverse locations. Thus, in the pay zone, the Boingo platform is a variety network for paid users. The paid services are simply upgraded versions of the basic free service. Other enhanced plans involve different locations, different device limits, and different time limits. The locations that offer Boingo Wi-Fi also have to pay Boingo to install and maintain the Wi-Fi infrastructure, which provides Boingo with another source of revenue.

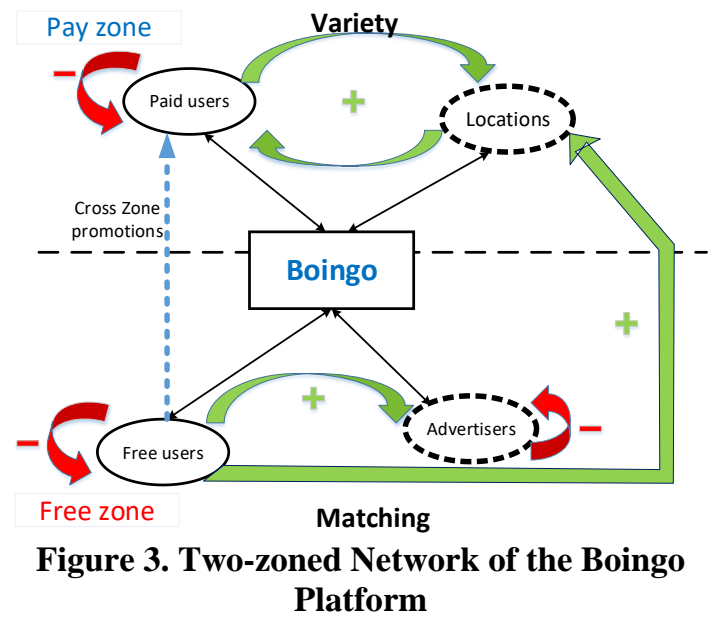

The second example is YouTube, which is a platform that connects users, content providers, and advertisers. We divide the YouTube network into two zones, as shown in Figure 4. Both free and paid users care about the quality and diversity of the content available on the platform. The main goal of YouTube users is to have access to as many interesting videos as possible, so the platform's function is to provide variety in both free and pay zones. Users also have cross-zone communications, as both free and paid users can communicate with and influence each other through comments, likes, dislikes, and subscriptions. In both free and pay zones, there are positive cross-side network effects between users and content providers. The more content providers who upload videos, the more users who want to access this platform. A larger user base also encourages more content providers to release videos on the platform. Within each group, users can communicate with each other about the content they watch, and content providers compete with one another for viewers and subscribers. One advantage of the $2 \mathrm{ZN}$ model is that it can handle more sophistication with the incorporation of zone. This additional dimension may be especially useful when analyzing market power and antitrust implications. It is noted that YouTube was acquired by Google in 2006. At that time, it was Google's second-largest acquisition. With numerous acquisitions like YouTube, Google has been the subject of the investigation by the Federal Trade Commission. Considerable debates among scholars and industry experts continue regarding the concern that the company is becoming a monopoly. Both government regulators and company executives can use the $2 \mathrm{ZN}$ model to further analyze acquisition cases like YouTube and make more convincing arguments. 
Next we analyze GitHub, which is a Web-based "git" repository hosting service platform that connects individual users working on projects. The free and pay zones of the GitHub network are depicted in Figure 5. Individual users have many opportunities to learn from and communicate with other experienced users about programming and other IT skills. Two different plans for GitHub users exist: the personal plan and the organization plan. The personal plan is for individuals looking to share their own projects and collaborate with others, while the organization plan is for businesses to manage teams using varying permission levels. In both plans, there are different subscription versions. Various charges not only provide users with different levels of public repositories but also create private repositories. Private repositories have limited visibility that is available only to their owners and collaborators, while public repositories are viewable to anyone. The number of private repositories allowed is determined by specific paid plans, which also offer different levels of additional services. The function of the GitHub platform is to provide connectivity and variety to both free and paid users in terms of communicating with others and learning from many different projects.

The 2ZN model sheds light on the dynamics of GitHub and can help the company self-analyze their exposure to antitrust. Microsoft has recently announced a plan to acquire GitHub. However, if the European Union (EU) Competition Agency raises serious concerns about the acquisition, it can launch a fullscale investigation to impede or delay the progress. The 2ZN model provides new legal angles, and it increases the transparency of important issues that need to be considered in the acquisition process by both regulators and the stakeholder companies.

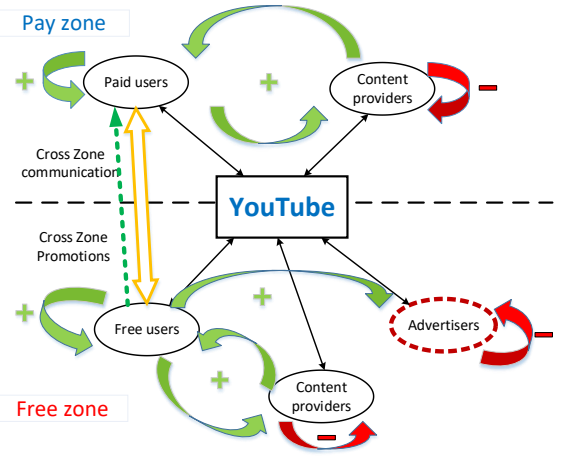

\section{Figure 4. Two-zoned Network of the YouTube Platform}

Finally, LinkedIn represents the most complex case among the four platforms for our analysis. As shown in Figure 6, both free and pay zones exist on the LinkedIn platform. In the free zone, people use LinkedIn as a professional social networking site. They develop and update their profiles to connect with other users. From this perspective, LinkedIn is similar to Facebook but more professionally oriented. Helping users maintain and grow their professional networks is the LinkedIn platform's primary function, which is used mainly by people who are not necessarily seeking jobs. Users update their CVs, connect with others in their fields and in working groups, become acquainted with key players, and develop their own reputations. In this light, the basic function of the platform is connectivity. There are also forum sections on LinkedIn. Users can follow the blogs of other users, content providers, established companies, and their friends to learn about current trends in an industry and gain insights on the future of a sector. In this role, LinkedIn is similar to Twitter, but the content posted is more businessfocused and professionally targeted.

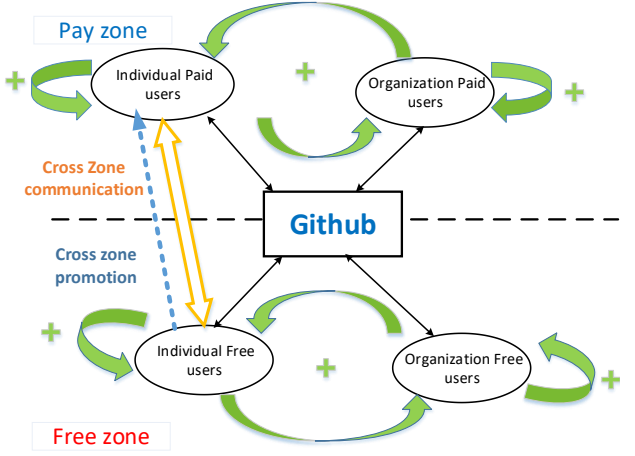
Figure 5. Two-zoned Network of the GitHub
Platform

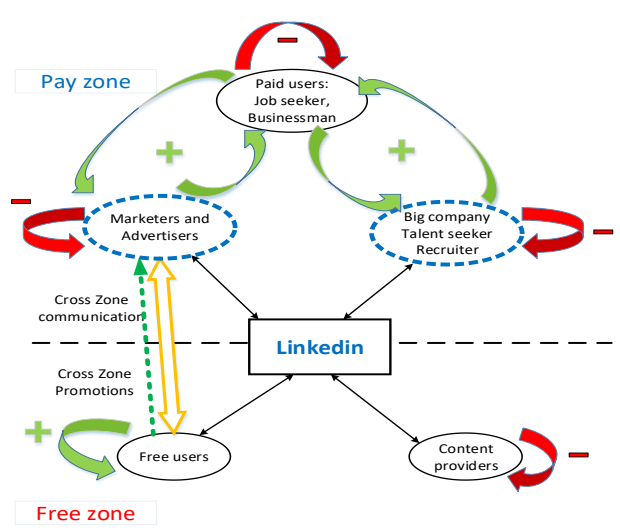

\section{Figure 6. Two-zoned Network of the LinkedIn Platform}

In the free zone, the basic social network functions of the website are sufficient for most users who access LinkedIn for networking and content consumption, but insufficient for other more specific tasks such as talent 
searches, sales opportunities, or job hunting. In other words, the basic services are not comprehensive enough for paid customers in the pay zone to carry out such tasks and achieve their goals. Paid users leverage LinkedIn to perform other additional tasks that are much more varied in scope. These premium subscriptions by individuals accounted for $20 \%$ of company revenues in 2011. There are four different LinkedIn premium accounts for individuals: Job Seekers, Business Plus, Sales Navigator Professional, and Recruiter Lite. These target potential employees, businesses, salespeople, and recruiters, respectively. Additional functions and features are provided with these four premium accounts.

Apparently, users in the pay zone differ from users in the free zone as each group uses different functions and features of the platform. Job seekers and recruiters look for each other through LinkedIn's matching function, which helps a job seeker identify companies that are looking for certain expertise and skills. Businesses looking for sales opportunities can also search for potential interested buyers. In addition, premium account users enjoy higher quality services than free users. When a user performs a search, for example, profiles of premium members appear on the first page of the results. With regard to technical support, premium users receive a faster response than those who do not pay. In other words, LinkedIn provides paid users with better services. In contrast, users in the free zone access the platform mainly to connect with others and view a variety of available content. Finally, because of the differences in mechanisms between the pay zone and the free zone, network effects are also shown to manifest differently.

As for the main functions of the two-sided networks in the two zones, they are quite different and hence LinkedIn exhibits a low level of zone parallelism. In the free zone, LinkedIn serves as a social networking platform for the purpose of connectivity and variety. For most free users, LinkedIn is used to connect with other professionals in order to build and grow their social networks. For content providers and viewers, the function of LinkedIn is to provide a rich variety of content. In the pay zone, for salespeople and business customers as well as for job hunters and recruiters, LinkedIn functions as a matching platform that assists members of two distinct groups with heterogeneous needs in seeking out each other for business transactions or employment opportunities.

LinkedIn was acquired by Microsoft in December 2016. Just like the case of Microsoft attempting to acquire Github, both companies could analyze their own market power and potential antitrust arguments using the proposed $2 \mathrm{ZN}$ model. There are multiple sides, which involve several distinct groups and indirect network effects. At a higher level, there are two zones that lead to cross-zone network effects. The traditional monopoly tests (e.g., SSNIP/HM) only consider one service in a user group and may be insufficient for the cases like LinkedIn. The $2 \mathrm{ZN}$ model provides constructs for new types of monopoly tests for these complex platforms. For example, an app store that provides music, video and podcasts might be monopolistic if it is deemed that $2 \mathrm{ZN}$ attributes are leveraged to eliminate competition. Separating music, podcasts and videos into three distinct $2 \mathrm{SNs}$ exemplifies a platform owner's decision to mitigate the risk of regulatory antitrust action.

\section{Attributes of Two-Zoned Networks}

In this section, we discuss the use of three attributes to help differentiate two-zoned networks. The first attribute, zone parallelism, assesses the functional similarity between the two zones. Focusing on the pay zone, the second attribute, service diversity, examines the variety in both extent and types of paid services provided in the pay zone. The third attribute, latent payment, looks at whether the free services provided in the free zone are truly free, or whether they incur any opportunity costs to the free users.

\subsection{Zone parallelism}

We define zone parallelism as the extent of functional similarity between the two zones in a twozoned network. In Eisenmann's [5] model of two-sided markets, platforms can be categorized into connectivity, variety, matching, or price setting, based on their functions. The level of zone parallelism varies across the four two-zoned networks that are discussed in the previous section. Figure 7 shows where they belong along this dimension of zone parallelism. YouTube has the highest zone parallelism because the platform performs the same function of providing variety in both the free zone and the pay zone. On the other hand, LinkedIn exhibits the lowest zone parallelism, as the platform provides connectivity and variety in the free zone while it matches job seekers and recruiters as well as sales people and businesses in the pay zone. Finally, GitHub and Boingo are situated in the middle of the spectrum. Zone parallelism has significant implications for the platform ecosystem. For instance, it can help platform owner decide how to balance the investments and resources between the two zones. It can also guide platform designer toward a better design plan for the infrastructure. In addition, in circumstances where zone parallelism is purposefully manipulated in order to maintain a monopolistic competitive position, legal implications become more 
transparent and actionable by examining the platform through this attribute.

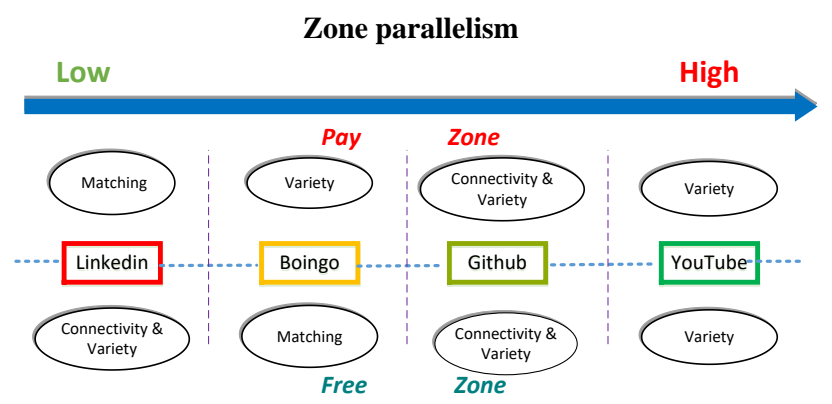

Figure 7. Zone Parallelism of Two-zoned Networks

\subsection{Service diversity for paid users}

In the pay zone, the platform provides different levels and/or varieties of services for paid users, and these diverse services are reflected in different prices. These paid services are either extended or upgraded versions of the basic services, and the main goal is to "extract" profits from the user base that already exists. We consider two aspects in describing various paid services. One is the extent to which the paid services are an enhanced version of the basic free services (i.e., whether the platform essentially provides the same services, albeit with differing quality, length, or number of devices and locations). This extent aspect of attribute has implications for the design and investment in the infrastructure. According to tenets of service computing, the infrastructure design should be consistent with the "service" elements. Boingo, for example, provides different lengths of service time, and the service extent also differs in the number of devices that customers can use to connect to Boingo Wi-Fi. Some plans allow up to two mobile devices, some allow any two Wi-Fi devices, and others allow any four $\mathrm{Wi}-\mathrm{Fi}$ devices. While the prices reflect the different extents of services, the nature of such services remains the same.

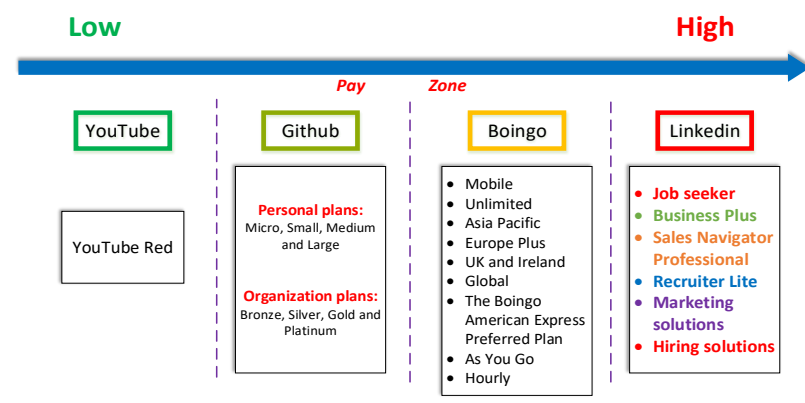

Figure 8. Diversity of Services for Paid Users in Two-zoned Networks
The second aspect we consider for service diversity reflects the variety in the added services provided in the pay zone. Some two-zoned networks offer additional paid services that target different market segments. One representative case is LinkedIn, which provides four different plans for paid users. The 'Job Seekers' plan targets users who wish to look for employment opportunities through LinkedIn, so this plan has features that help a user stand out from other job seekers to hiring managers. The 'Business Plus' plan is aimed at customers who desire to promote and grow their businesses. Therefore, this plan has features that help users maximize the reach of their networks. The 'Sales Navigator Professional' plan targets people who intend to seek sales opportunities. Finally, the 'Recruiter Lite' plan is intended for those who wish to quickly identify qualified candidates suitable for their open positions. This plan provides such functions as automatic candidate tracking, recruiting-specific design, and integrated hiring. In a decision on the merger of Microsoft and LinkedIn, a service diversity issue was raised in the EU regarding the nature of the data that LinkedIn had amassed and how that data might be leveraged to deliver, for example, additional, anti-competitive Microsoft services. The EU decided that the data-enabled services would not create antitrust issues because a large amount of Internet data is readily available for competitors to leverage in the creation of a diverse set of potentially competing services.

As shown in Figure 8, LinkedIn has the highest diversity because the platform offers the greatest number of paid service types, each with a different function, as described above. On the other hand, YouTube shows the lowest diversity, as the platform provides only one paid service (YouTube Red), which is essentially the enhanced version of the free service without commercials. Finally, Boingo and GitHub show moderate levels of diversity in the pay zone.

Service diversity highlights the fact that different infrastructure costs can be incurred in supporting different levels of services across zones. These costs can impact technical choices where some architectural approaches may be deemed more desirable than the others. This construct may matter the most to the infrastructure side of the platform design. This attribute can also enhance the ability to bundle services that aims to stave off competition in conducting improper competitive behavior. The design of the underlying architecture of a platform can manifest the intent of platform designers as they evolve the platform for anticipated competition. As such, service diversity provides important insights to those studying platform market issues as well as a construct that can be crucially relevant in antitrust policy debates. 


\subsection{Latent payment by free users}

Like the customers in the pay zone who receive additional services by making direct cash payments, users in the free zone are sometimes required to make latent payments. Boingo and YouTube users, for example, typically are asked to watch a mandatory video commercial before they can access the main services. In such cases, customers pay indirectly with their time for the free services they enjoy on the platform. This practice of latent payment is related to the concept of opportunity cost in that if people want to consume something (e.g., Wi-Fi), they have to give up something else (e.g., their time). In the free zone, users "pay with their time" so while they do not make a direct cash payment, they make a latent payment. Therefore, the requirement that users in the free zone make such a latent payment is another attribute that distinguishes two-zoned networks. In recent antitrust actions, the ability of a platform owner to manipulate latent payments is seen as a possible way to temporarily reduce customer 'costs' in circumstances where a rival digital advertising business mounts a challenge for a particular business segment. Using latent payment analysis can expose such potential manipulation, thereby enabling more informative regulatory action. Overall, two of the four cases we examined above require latent payment (Boingo and YouTube) while the other two do not (GitHub and LinkedIn).

In summary, the three attributes of zone parallelism, service diversity, and latent payment for the four two-zoned networks are shown in in Table 1. According to the above discussion, the four two-zoned networks differ across these three attributes. LinkedIn has a low level of zone parallelism, displays high diversity of additional services in the pay zone, and requires no latent payment of its users in the free zone. Boingo has a low level of zone parallelism, shows medium to high diversity of additional services in the pay zone, and requires its free-zone users to make latent payment. GitHub, on the other hand, has a high level of zone parallelism, exhibits medium to low diversity of additional services in the pay zone, and does not require its free-zone users to make latent payment. Finally, YouTube has the highest level of zone parallelism but the lowest diversity of additional services in the pay zone, and its users have to make latent payment when using the free service. These examples along with their respective attribute analysis demonstrate that the $2 \mathrm{ZN}$ approach is more comprehensive than the 2SN approach, and in our discussion of each $2 \mathrm{ZN}$ attribute, we show relevance to practical and highly timely legal issues being addressed across the globe.

\begin{tabular}{|l|c|c|c|}
\hline & $\begin{array}{c}\text { Zone } \\
\text { Parallelism }\end{array}$ & $\begin{array}{c}\text { Service } \\
\text { Diversity }\end{array}$ & $\begin{array}{c}\text { Latent } \\
\text { Payment }\end{array}$ \\
\hline LinkedIn & Low & High & No \\
\hline Boingo & Low & Medium-High & Yes \\
\hline GitHub & High & Medium-Low & No \\
\hline YouTube & High & Low & Yes \\
\hline
\end{tabular}

Table 1. Summary of the Three Attributes for the Four Two-zoned Networks

\section{Conclusion}

In this paper, we propose a theoretical model of two-zoned network to complement the extant literature of two-sided networks. We use four real-world cases to illustrate its applications. In the proposed model, we introduce a new dimension of "zone," which extends the existing dimension of "side" in the two-sided network. The core platform provides two types of services: free and paid, in each corresponding zone. A conventional two-sided market exists in each zone. The core platform not only serves as a nexus of the two zones but also connects different user groups in each zone, rendering it both strategically and operationally important. Three specific attributes are identified to help illuminate the structure of two-zoned networks: zone parallelism, diversity of services in the pay zone, and latent payment in the free zone. The four cases are shown to exhibit noticeable differences in the three attributes.

The necessity of splitting a network into two zones is manifested in the different values the platform provides to different groups of users in each zone. As such, varying perspectives and rules are needed to devise the strategy and technology development plans a platform should implement for each zone. In order to do so, platform owners should know the structure of their network platforms and resultant markets. Our two-zoned network model helps achieve this goal by complementing the traditional model of two-sided network to fill both the gap in literature and the deficiency in practice.

Theoretically, our proposed model of two-zoned network contributes to the extant literature of $2 \mathrm{SN}$. There are cases in which the traditional 2SN model does not cover every aspect of network structure. The three attributes that we propose also shed light on this new type of platform-mediated networks. Practically, our study helps platform owners better understand the ecosystem of their two-zoned networks. Competition today is much more dynamic and volatile, and a single strategic mistake can doom a platform's future. Getting a clearer picture of its market structure and a better understanding of its interaction mechanism is thus 
crucial. The issues of pricing decisions, the degree of openness, actions to cope with the "winner-take-all" outcome, and ways to avoid envelopment threat should all be considered when using the new $2 \mathrm{ZN}$ model to devise competitive strategy.

The 2ZN model also provides new perspectives and insights to the legal issue of antitrust. The traditional tools (e.g., SSNIP/HM tests) for measuring the market power of a platform need to be modified and enhanced to consider the existence of additional zones beyond just sides. The ability to exercise market power on one zone of a platform very likely depends on the structure of the other zone. For instance, in the presence of rarely studied cross-zone network effects, users in one zone of the platform can be viewed as inputs to the supply of services for users in the other zone. Even if the concentration of power in one zone of the market is not strong enough to enable a dominant position in that zone, it may still be relevant as it can firstly increase the platform's negotiating power and then increase prices in the other zone where a company can take advantage of its existing market power. In this light, zone parallelism can be especially useful in identifying ways to complement SSNIP/HM tests. Both plaintiffs and defendants in such antitrust cases of platform competition should consider the user groups and services for each side in each zone in order to make solid inferential justifications.

Interesting questions for future research on the topic of two-zoned network can be identified. Firstly, if archival data about the cases examined in the paper become available, one can investigate relevant issues using econometric models to validate the empirical applicability of the two-zoned network. Secondly, an analytical study can be conducted to model the behaviors of different agents, such as owners, providers and users, in the two-zoned network. Thirdly, researchers are encouraged to look at other companies that also can be examined using this new 2ZN model to compare findings and derive new implications. Other related issues include: whether providing free and premium account services will create more value for the platform; whether the platform can use certain strategies to encourage communication between different zones so as to create cross-zone network effects; and how the service variety in the pay zone can influence the utility of users, platform provider, and platform owner. These representative research questions are just a few of many that can further enhance our understanding of two-zoned networks.

\section{References}

[1] Bakos, Y., and Katsamakas, E. 2008. "Design and Ownership of Two-Sided Networks: Implications for Internet Platforms," Journal of Management Information Systems (25:2), pp. 171-202.

[2]Caillaud, B., and Jullien, B. 2003. "Chicken \& Egg: Competition among Intermediation Service Providers," RAND Journal of Economics, pp. 309-328.

[3] Clements, M. T., and Ohashi, H. 2005. "Indirect Network Effects and the Product Cycle: Video Games in the US, 1994-2002," Journal of Industrial Economics (53:4), pp. 515-542.

[4] Delrahim, M. 2019. "I'm Free: Platforms and Antitrust Enforcement in the Zero-Price Economy," Prepared for Delivery at Silicon Flatirons, University of Colorado Law School .

[5] Eisenmann, T. R. 2006. Platform-Mediated Networks: Definitions and Core Concepts.

[6] Eisenmann, T. R., Parker, G., and Van Alstyne, M. W. 2008. "Opening Platforms: How, When and Why?," Platforms, markets and innovation, pp. 131-162.

[7] Gawer, A. 2014. "Bridging Differing Perspectives on Technological Platforms: Toward an Integrative Framework," Research Policy (43:7), pp. 1239-1249.

[8] Hoppner, T., 2015. Defining Markets for Multi-Sided Platforms: The Case of Search Engines. World Competition, (38:3), pp.349-366

[9] Katz, M., and Sallet, J., 2018. "Multisided Platforms and Antitrust Enforcement," Yale Law Journal, (127:7), pp. 2142-2175

[10] Khan, L. M. 2017. “Amazon's Antitrust Paradox," Yale Law Journal, (126:3), pp. 710-805.

[11] Moresi, S. X., Salop, S. C., and Woodbury, J. R. 2008. "Implementing the Hypothetical Monopolist SSNIP Test with Multi-product Firms," Antitrust Source, Retrieved from: https://papers.ssrn.com/sol3/papers.cfm?abstract id=108753 $\underline{6}$

[12] Morton, Scott, F., and Nierenberg, T. 2019. "Report: Committee for the Study of Digital Platforms-Market Structure and Antitrust Subcommittee." George J. Stigler Center for the Study of the Economy and the State, The University of Chicago Booth School of Business.

[13] OECD, 2018. Rethinking Antitrust Tools for MultiSided Platforms.

[14] Parker, G.G., Van Alstyne, M.W. and Choudary, S.P., 2016. Platform Revolution: How Networked Markets Are Transforming the Economy and How to Make Them Work for You. WW Norton \& Company.

[15] Pierce, L. 2009. "Big Losses in Ecosystem Niches: How Core Firm Decisions Drive Complementary Product Shakeouts," Strategic Management Journal (30:3), pp. 323347.

[16] Rochet, J.-C., and Tirole, J. 2003. "Platform Competition in Two-Sided Markets," Journal of the European Economic Association (1:4), pp. 990-1029. 